\title{
Evaluating the effect of mineralogy and mechanical stability of recycled excavation materials by Los Angeles and micro-Deval test
}

\author{
S. Adomako, R.T. Thorstensen, N. Akhtar \& M. Norby \\ Department of Engineering and Science, University of Agder, Grimstad, Norway \\ C.J. Engelsen \\ Department of Building and Infrastructure, SINTEF Community, Oslo, Norway
}

T. Danner

Department of Architecture, Materials and Structures, SINTEF Community, Oslo, Norway

D.M. Barbieri

Department of Civil and Environmental Engineering, Norwegian University of Science and Technology,

Trondheim, Norway

\begin{abstract}
This paper presents the findings of the mechanical performance of recycled excavation materials (REM). The aim was to document the stable performance of REM and to suggest its suitability for unbound applications. Test operations were performed at different schedules with the Los Angeles (LA) and micro-Deval (MD) test machine. X-ray diffraction (XRD) analysis was performed to examine the effect of mineralogy on the performance of REM. The results showed that REM showed consistent LA performance, but significant variations were observed in MD values. XRD results showed that up to $20 \%$ of chlorite and mica minerals had no influence on the LA performance of REM.
\end{abstract}

Keywords: Recycled excavation materials, Los Angeles test, micro-Deval test, Mica, Chlorite

\section{INTRODUCTION}

Recycled excavation materials (REM) present many opportunities in the construction industry. The substitution of conventional and non-renewable construction materials with stabilized REM is gaining global interest due to the environmental, economic, and technical performance. Regarding material substitutions, significant factors such as the type of application, geological properties, conformity with technical requirements (Barbieri et al. 2019; Arulrajah et al. 2012; Sasanipour and Aslani 2020), and location, are mainly considered.

The transformation process of natural ores including weathering is considered a significant parameter in determining the relationship between geological properties and mechanical performance. Considering mineralogy, distinct variations exist in the mineral properties of the same nomenclature of aggregates such as limestones, dolomites and quartzites (Gehringer et al. 2012). REM in general is characterised by a wide range of compositional and geological properties. Hence, uniformity of mechanical performance within the same source or from different sources is not always achieved. In addition, the literature does not provide comprehensive documentation of Los Angeles (LA) and micro-Deval (MD) values of REM to 
demonstrate the variations or stabilization of strength properties given the possibility that soft rocks i.e., mudstone, sandstone, conglomerate rock etc. could be a composition of REM. Soft rocks are characterized by low engineering properties and certain geological characteristics e.g., poor cementation, weak intergranular boundaries, clayey minerals etc.

Regarding the effect of mineral composition on strength properties of rocks, the literature present unique findings based on the type and formation of mineral group. Considering primary minerals, it was reported in a review by Adomako et al. (2021), that quartz and feldspar largely contributed to the LA and MD performance of rocks, and generally, about $20 \%$ of soft minerals (mica) had no significant effect on the performance of rocks. Furthermore, the spatial distribution of grain size and shape were important factors considered for overall assessment of the performance. Ajagbe et al. (2015) mentioned that increased content of quartz improved the LA resistance of metamorphic rocks. The study by Pang et al. (2010) and Afolagboye et al. (2016) showed that increased content of quartz or feldspar, or a proportional ratio of both resulted in high resistance to LA. The study by Ademila (2019) also showed the relationship between LA of granite gneiss and granite rocks and the content of quartz and feldspar. Regarding quartzite rocks, the same study reported low LA performance although quartzite had high amount of quartz with insignificant amount of feldspar.

In other mechanical test, Hemmati et al. (2020) studied the effects of mineralogy and textural characteristics on compressive and tensile strengths of crystalline igneous rocks. Their study found that the quartz to K-feldspar size ratio was the major indicator that influenced the performance. The differences in connection to mineralogy on each strength property showed that K-feldspar influenced the tensile strength and plagioclase, and quartz influenced the compressive strength. In the case of granitic rocks, Sousa (2013) mentioned that large amount of feldspar increases strength property while quartz resulted in cracking due to its low capacity to withstand stress. Conversely, Tuğrul and Zarif (1999) reported that quartz had low or no cleavage planes and therefore it had the tendency to improve strength while feldspar reduced the strength property of aggregates.

Mica minerals (biotite and muscovite) represent a group of sheet silicates with basal cleavage characteristics and are mainly formed by activities of chemical weathering. There is a strong relationship between the content of mica minerals and mechanical performance (Tandon and Gupta 2013). Given the amount of mica, the study by Anastasio et al. (2016) and Fortes et al. (2016) reported that about $20 \%$ of mica does not compromise the strength performance of meta-greywacke. In addition, both studies reported that sparkle traces of mica found in amphibolite did not affect the performance. Nålsund (2010) also showed that $20 \%$ of mica content could not be classified to have a negative influence on the performance of rocks. According to Amuda et al. (2014) the probable cause to high resistance to LA in porphyritic granite was the low content of mica. Similarly, the study by (Solomon et al. 2021, manuscript in preparation) found that a positive relationship between mica and the LA of porphyritic granite. Another important consideration given to the effect of mica is the distribution and structure of the grain boundaries (Fortes et al. 2016).

This study was aimed at evaluating the stability of LA and MD performance of REM and to investigate the effect of soft minerals on the performance. The REM samples varied interms of composition. Natural aggregates (NA) were included in the experimental study in order to compare the results. Test operations were performed at different schedules. The results of the study may increase the confidence to apply REM in unbound construction.

\section{METHODOLOGY}

\subsection{Sample preparation}

Velde Pukk operates an innovative and sustainable recycling plant (CDE Aggmax TM system) for the production of REM. Table 1 below describes the REM and NA used in the study. In total, 25 batches of REM and NA were collected from the operational plant within the period of $09 / 10 / 2019$ to $05 / 12 / 2019$. 18 samples represented REM and 7 consisted of $100 \%$ NA. 
Batched samples weighed 22-50 $\mathrm{kg}$ and were tested following the dates 09/10/2019 - 17/10/ 2019 (Batch 1), 29/10/19 -06/11/19 (Batch 2) and 19/11/19 - 05/12/19 (Batch 3). Both REM and NA showed no visible coatings.

Table 1. Description of REM and NA samples used in the experimental study.

\begin{tabular}{lllll}
\hline $\begin{array}{l}\text { Sample } \\
\text { name }\end{array}$ & Type of material & Processing & Source & $\begin{array}{l}\text { Particle-Size } \\
\text { (d/D) mm }\end{array}$ \\
\hline REM & Excavation material & $\begin{array}{l}\text { Separation, washing \& } \\
\text { fractionation } \\
\text { Two-three stage crushing }\end{array}$ & $\begin{array}{l}\text { Velde Pukk } \\
\text { (Sandnes) }\end{array}$ & $\begin{array}{l}\text { Velde Pukk } \\
\text { (Sandnes) }\end{array}$ \\
NA & Crushed & $8 / 16$ \\
\hline
\end{tabular}

\subsection{Samples used for $X$-ray diffraction (XRD) analysis}

Table 2 below shows the composition of fractions obtained for XRD analysis after LA tests. Three samples of REM and two NA were selected. The XRD analysis was performed on pulverized fraction i.e., passing the $1.6 \mathrm{~mm}$ sieve after LA. This approach helped to establish concrete relationships between the disintegration effect of LA on samples composed of soft minerals.

Table 2. Composition of samples obtained for XRD analysis.

\begin{tabular}{|c|c|c|c|c|c|c|c|}
\hline \multirow[b]{2}{*}{$\begin{array}{l}\text { Sample } \\
\text { name }\end{array}$} & \multirow[b]{2}{*}{$\begin{array}{l}\text { Fraction for } \\
\text { XRD after LA }\end{array}$} & \multicolumn{6}{|l|}{ Composition } \\
\hline & & $\begin{array}{l}\text { Granite/ Gneiss/ Quartzite/ } \\
\text { Feldspathic rock }\end{array}$ & Phyllite & $\begin{array}{l}\text { Dark } \\
\text { rock }\end{array}$ & Asphalt & Mortar & $\begin{array}{l}\text { Light- } \\
\text { weight } \\
\text { particles }\end{array}$ \\
\hline NA-4 & $<1.6 \mathrm{~mm}$ & 100 & & & & & \\
\hline NA-5 & $<1.6 \mathrm{~mm}$ & 100 & & & & & \\
\hline REM-5 & $<1.6 \mathrm{~mm}$ & 71 & 25 & 2 & 2 & & \\
\hline REM-6 & $<1.6 \mathrm{~mm}$ & 91 & 6 & & 1 & 1 & 1 \\
\hline REM-13 & $<1.6 \mathrm{~mm}$ & 98 & 2 & $<1$ & & & \\
\hline
\end{tabular}

\subsection{Physical and mechanical test}

The particle-size distribution (PSD) was based on the procedure described in NS-EN 933-1.

The LA test measures the fragmentation resistance of construction materials. The CEN1097-2 specifies particle size of $10 / 14 \mathrm{~mm}$ for the test. A test mass of $5000 \pm 5 \mathrm{~g}$ was poured into cylindrical drum and eleven steel balls were gently added to the test mass. One complete test cycle was 500 revolutions of the steel drum at 31-33 revolutions per minute. Afterwards, the tested mass was washed on a $1.6 \mathrm{~mm}$ sieve, oven dried to a temperature of $(110 \pm 5){ }^{\circ} \mathrm{C}$ and the mass loss $(\%)$ was determined.

The MD test was performed following the description in CEN-1097-1. The particle size for MD test was $10 / 14 \mathrm{~mm}$ and mass of the test sample was $500 \mathrm{~g}$. Spherical steel balls of 5000 $\mathrm{g}$ in weight was added to the test mass and $2.5 \pm 0.5 \mathrm{~L}$ of water was gently poured into each drum containing the specimen. Each test cycle was up to 2000 revolutions and after the test, 
the tested sample was washed on $1.6 \mathrm{~mm}$ sieve, dried in the oven and the average loss of mass $(\%)$ for two test specimens was calculated as the micro-Deval coefficient.

\section{RESULTS AND DISCUSSION}

\subsection{Physical and mechanical performance}

In Figure 1, the gradation curves shown represent the materials used for the XRD analysis. Attempt was made to evaluate the dense composition of the samples after LA, and which sample had highly pulverized particles. Comparing the PSD before and after LA, it was observed that pulverized test portions after LA showed that REM- 6 had about $9 \%$ of $<4 \mathrm{~mm}$ fraction while all other samples had about $5 \%$ below $4 \mathrm{~mm}$. In addition, the pulverization degree followed a systematic pattern. This was confirmed by the mass retained on $<1.6 \mathrm{~mm}$ sieves measured for LA coefficient.
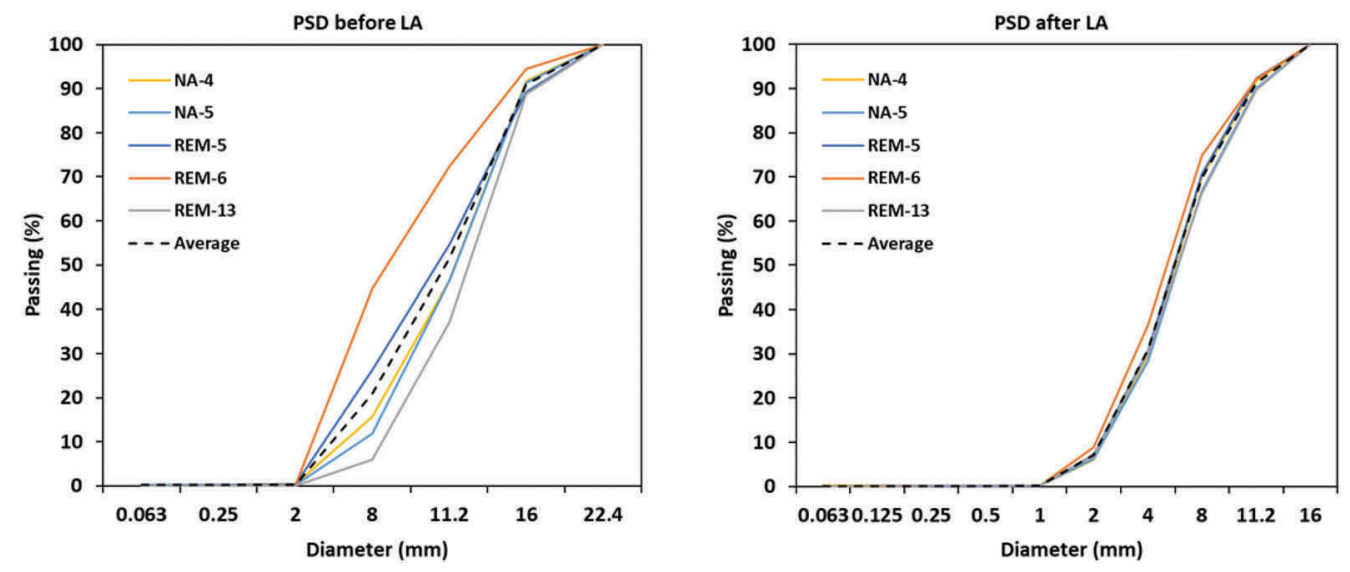

Figure 1. PSD of REM and NA samples before and after LA.

Regarding the LA and MD of REM and NA, Figure 2, Figure 3 and Figure 4 shows the performance of the samples in each batch. For the MD, simultaneous test operations were carried out at Velde following the same protocol of batch number and test dates (Velde AS, 2020). The LA and MD threshold applied on base and subbase materials in Norway were used as reference (NPRA, 2014).

In Figure 2, consistent LA values of $23 \%$ and $6 \%$ for MD were observed in both NA-1 and NA-2. The LA values of REM were very close. REM-1 recorded $28 \%$, REM-2 and REM-4 showed the same LA performance of $27 \%$ and REM-3 had $26 \%$.

Considering the MD, REM-1 recorded the highest at $20 \%$, followed by REM- 2 at $15 \%$. Both REM-3 and REM-4 had 9\% and 7\%, respectively, showing a high resistance to abrasion. While the LA limit requirement was met by all REM samples, REM-1 did not meet the MD threshold.

Figure 3 shows the LA and MD performance for samples in batch 2. The LA values for NA-4 and NA-5 varied at $27 \%$ and $22 \%$ respectively. However, the MD did not show any significant variation between them; NA-4 had MD of $8 \%$ and NA-5 showed $6 \%$ MD value.

Regarding REM, REM-5, and REM- 8 had the same LA of $25 \%$. REM- 6 recorded the highest LA at 28\% amongst the REM received in batch 2. REM-7 and REM-9 also had LA of $24 \%$ in each case and REM-10 had LA of $26 \%$. REM in this batch met the LA criteria. 


\section{Batch 1}

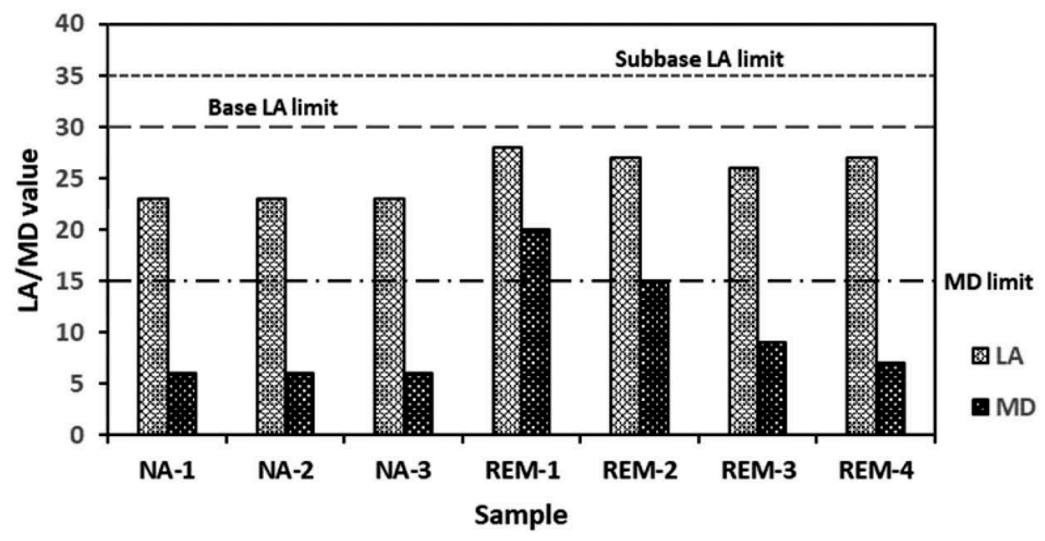

Figure 2. LA and MD performance of batch 1 samples received on 09/10/2019 - 17/10/2019.

For MD values of REM, REM-5 had MD of 17, being the highest amongst the studied REM in this batch. REM-6, REM-7 and REM-9 had the same MD of 10\%. REM-8 and REM-10 had MD of 9 and 7, respectively. Only REM-5 exceeded the MD threshold.

\section{Batch 2}

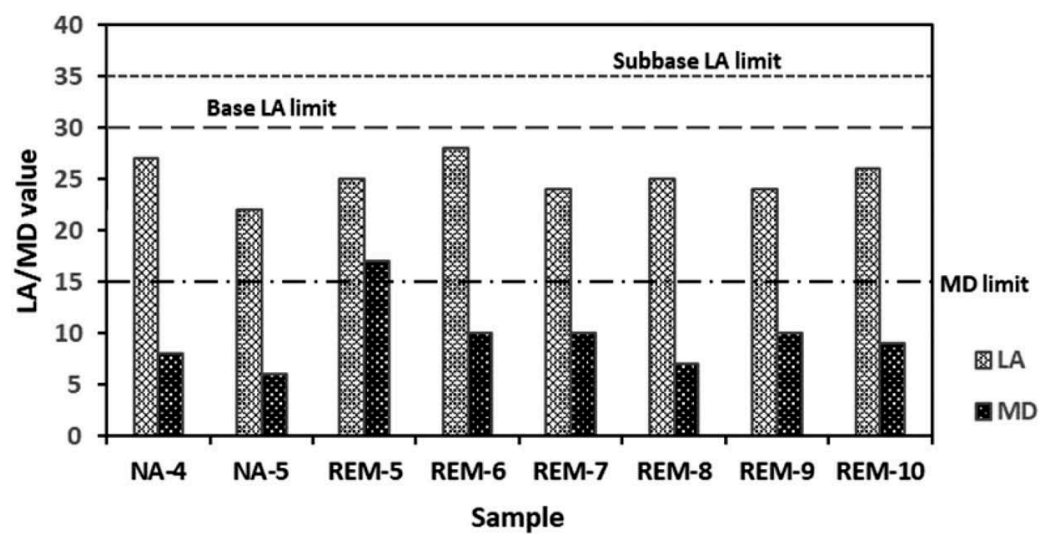

Figure 3. LA and MD performance of batch 2 samples received on 29/10/19 - 06/11/19.

Figure 4 shows the performance of batch 3 samples. NA-6 and NA-7 had LA of $29 \%$ and $24 \%$ respectively. MD test on NA-6 was not performed. However, NA-7 had a good MD of $6 \%$. Accordingly, it could be assumed that the MD performance of NA-6 would not significantly vary from NA-7.

For REM samples, close LA values was observed. REM-11, REM-12 and REM-16 had $26 \%$ LA value. REM-13 and REM-15 also had $24 \%$ of LA, and $25 \%$ of LA was recorded for both REM-14 and REM-17. The LA of REM-18 was 27\%.

Regarding the MD of REM, both REM-11 and REM-13 showed the same resistance to abrasion with MD of 10\%. REM- 12 and REM-14 also showed MD of $11 \%$. Both REM-15 
and REM-17 had the same MD of 8\%. The MD for REM-16 was 9\% and REM-18 produced MD of $12 \%$. In batch 3, the LA and MD performance met the threshold.

\section{Batch 3}

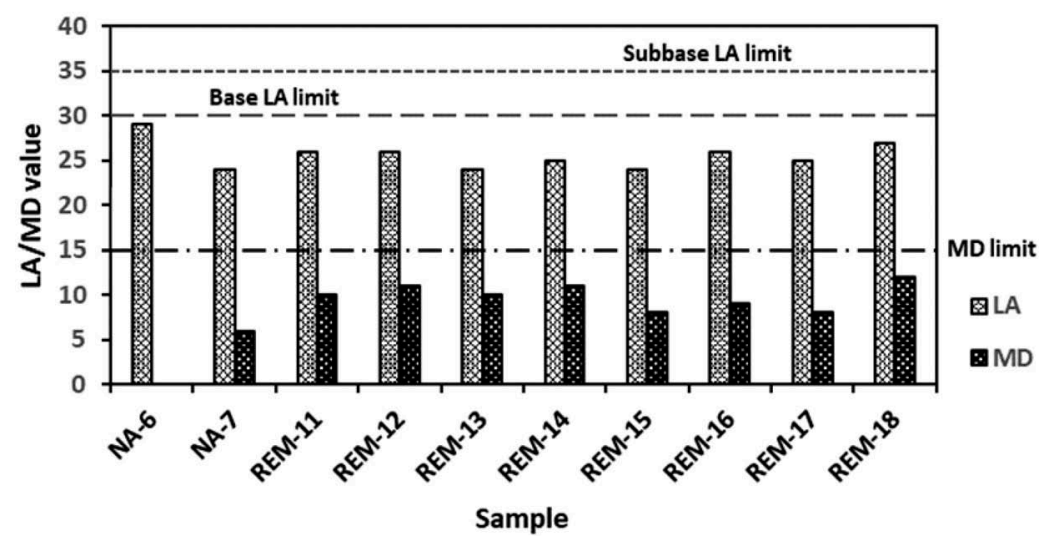

Figure 4. LA and LA and MD performance of batch 3 samples received on 19/11/19 - 05/12/19.

\subsection{Relationship between LA and mineralogy of REM and NA}

In Figure 5, the mineral composition of pulverized samples of REM and NA obtained from < $1.6 \mathrm{~mm}$ sieves after LA test is shown. The XRD results showed high peaks of quartz present in all the samples. In addition, significant peaks of mica and chlorite was found in REM-5 and REM-13. The amount of quartz present in all samples ranged from 34-38\%. Other minerals such as albite, microcline and anorthite of feldspar group were also present but not displayed in Figure 5. The connection between the low resistance to abrasion by MD in REM-5 could be explained by the content of both mica and chlorite. In Table 2, 25\% phyllite materials formed a component of REM-5 and 2\% in REM-13.

According to Razouki and Ibrahim (2019) the degree of abrasion of rock samples is connected to the hardness value measured by Mohs hardness scale. Mica and chlorite are phyllosilicates with low hardness value ranging from 2-4. Interms of LA, the difference between REM-5 and REM-13 was not significant. Previous findings (Anastasio et al. 2016, Fortes et al. 2016 and Nålsund 2010) have suggested that $20 \%$ of mica content does not significantly reduce the strength performance i.e., LA. The results of this study was consistent with the findings.

\section{CONCLUSIONS}

This study investigated the stability of mechanical performance of 18 recycled excavation materials (REM) and compared the performance to 7 natural aggregates (NA) samples. In addition, the mineralogy of REM and NA were analyzed to draw final conclusions on the relationship between the strength and mineralogy. The Los Angeles (LA) and micro-Deval (MD) test methods were used to evaluate the strength properties and X-ray diffraction analysis was performed on pulverized samples $<1.6 \mathrm{~mm}$ obtained after LA test.

Generally, the findings showed consistent LA performance of REM, but wide variations of MD performance was observed. The LA values of REM met the limit criteria for base and 


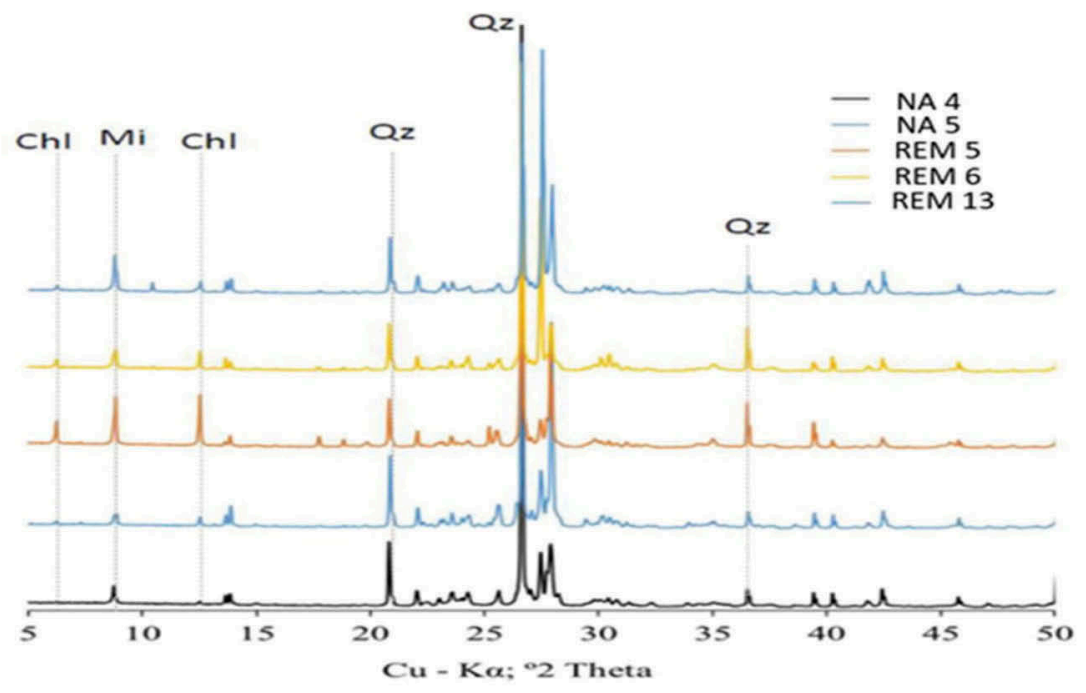

Figure 5. XRD results showing mineral peaks of $<1.6 \mathrm{~mm}$ fraction of REM and NA. Chl, Chlorites; Mi, Mica; Qz; Quartz are the dominant minerals shown.

subbase layers according to Norwegian Public Roads Administration Handbook (N200). Regarding the MD, REM-1 and REM-5 samples exceeded the threshold.

The results for mineralogy showed that REM-5 contained about $20 \%$ of chlorite and mica, however, the LA performance was not influenced. Perhaps, the low MD resistance of REM-5 could be attributed to the content of these weak minerals. This study contributes to establish the stable performance of REM and to raise the confidence of end users about the technical benefits.

\section{ACKNOWLEDGEMENTS}

The work presented in this paper is part of the ongoing project MEERC (More Efficient and Environmentally friendly Road Construction), partly funded by the Research Council of Norway (NFR) [project number 273700] and Sorlandets Kompetansefond. In addition, thanks to Velde Pukk for the MD test values.

\section{REFERENCES}

Barbieri, D.M., Hoff, I. and Mørk, M.B.E., 2019. Innovative stabilization techniques for weak crushed rocks used in road unbound layers: A laboratory investigation. Transportation Geotechnics, 18, pp.132 - 141. https://doi.org/10.1016/j.trgeo.2018.12.002

Arulrajah, A., Ali, M.M.Y., Piratheepan, J. and Bo, M.W., 2012. Geotechnical properties of waste excavation rock in pavement subbase applications. Journal of materials in civil engineering, 24(7), pp.924 - 932 .

Sasanipour, H. and Aslani, F., 2020. Durability properties evaluation of self-compacting concrete prepared with waste fine and coarse recycled concrete aggregates. Construction and Building Materials, 236, p.117540. https://doi.org/10.1016/j.conbuildmat.2019.117540

Gehringer, E., Read, D. and Tutumluer, E., 2012. Characterization of ballast performance in heavy axle loading (HAL). In Proceedings of AREMA 2012 Annual Conference.

Adomako, S., Engelsen C.J. and Thorstensen R.T, Barbieri D.M. Review of the relationship between aggregates geology and Los Angeles and micro-Deval tests. Bulletin of Engineering Geology and the Environment. 2021 Jan 12:1 - 8. 
Ajagbe, W.O., Tijani, M.A. and Oyediran, I.A., 2015. Engineering and geological evaluation of rocks for Concrete Production. LAUTECH Journal of Engineering and Technology, 9(2),pp.67-79. Retrieved from https://laujet.com/index.php/laujet/article/view/85

Pang, L., Wu, S., Zhu. J., Wan. L., 2010. Relationship between retrographical and physical properties of aggregates. Journal of Wuhan University of Technology-Mater. Sci. Ed. 25(4): 678-681. https://doi. org/10.1007/s11595-010-0069-0

Afolagboye, L.O, Talabi, A.O, Akinola, O.O., 2016. Evaluation of selected basement complex rocks from Ado-Ekiti, SW Nigeria, as source of road construction aggregates. Bulletin of Engineering Geology and the Environment 75(2): 853-865. http://doi.org/10.1007/s10064-015-0766-1

Ademila, O., 2019. Engineering geological evaluation of some rocks from Akure, Southwestern Nigeria as aggregates for concrete and pavement construction. Geology Geophysics and Environment 45(1): 31 43. http://doi.org/10.7494/geol.2019.45.1.31

Hemmati, A., Ghafoori, M., Moomivand, H. and Lashkaripour, G.R., 2020. The effect of mineralogy and textural characteristics on the strength of crystalline igneous rocks using image-based textural quantification. Engineering Geology, 266, p.105467.

Sousa, L.M., 2013. The influence of the characteristics of quartz and mineral deterioration on the strength of granitic dimensional stones. Environmental earth sciences, 69(4), pp.1333-1346. https://doi.org/ $10.1007 / \mathrm{s} 12665-012-2036-\mathrm{x}$

Tuğrul, A. and Zarif, I.H., 1999. Correlation of mineralogical and textural characteristics with engineering properties of selected granitic rocks from Turkey. Engineering geology, 51(4), pp.303-317. https://doi. org/10.1016/S0013-7952(98)00071-4

Tandon, R.S. and Gupta, V., 2013. The control of mineral constituents and textural characteristics on the petrophysical \& mechanical (PM) properties of different rocks of the Himalaya. Engineering Geology, 153, pp.125-143. https://doi.org/10.1016/j.enggeo.2012.11.005

Anastasio, S, Fortes A.P.P., Kuznetsova, E., Danielsen, S.W., 2016. Relevant Petrological Properties and their Repercussions on the Final use of Aggregates. Energy Procedia 97: 546-553. https://doi.org/ 10.1016/j.egypro.2016.10.073

Fortes, A.P.P, Anastasio, S, Kuznetsova, E, Danielsen, S.W., 2016. Behaviour of crushed rock aggregates used in asphalt surface layer exposed to cold climate conditions. Environmental Earth Sciences 75(21): 1414. https://doi.org/10.1007/s12665-016-6191-3

Nålsund, R., 2010. Effect of grading on degradation of crushed-rock railway ballast and on permanent axial deformation. Transportation Research Record 2154(1): 149-155. https://doi.org/10.3141/2154-15

Amuda, A.G. Uche, O.A.U. and Amuda A.K., 2014. Physicomechanical Characterization of Basement Rocks for Construction Aggregate: A Case Study of Kajuru Area, Kaduna, Nigeria. IOSR Journal of Mechanical and Civil Engineering 11(6): 46-51.

NS-EN 933-1: 2012 Tests for geometrical properties of aggregates - Part 1: Determination of particle size distribution - Sieving method, CEN_Standard_Norge, 201.

NS-EN 1097-2: 2010 Tests for mechanical and physical properties of aggregates. Part 2: Methods for the determination of resistance to fragmentation, CEN_Standard_Norge.

NS-EN 1097-1: 2011 Tests for mechanical and physical properties of aggregates Part 1: Determination of the resistance to wear (micro-Deval), CEN_Standard_Norge.

Velde AS, "Resultat LA og MDE," utg., 2020.

NPRA. Håndbok N200 Vegbygging. Norway: Vegdirektoratet;2014.

Razouki, S.S. and Ibrahim, A.N., 2019. Improving the resilient modulus of a gypsum sand roadbed soil by increased compaction. International journal of pavement Engineering, 20(4), pp.432-438. https://doi. org/10.1080/10298436.2017.1309190 